\title{
Multi-View Multiple Clusterings Using Deep Matrix Factorization
}

\author{
Shaowei Wei, ${ }^{1}$ Jun Wang,,${ }^{1, *}$ Guoxian Yu, ${ }^{1,2}$ Carlotta Domeniconi, ${ }^{3}$ Xiangliang Zhang ${ }^{2}$ \\ ${ }^{1}$ College of Computer and Information Sciences, Southwest University, Chongqing, China \\ ${ }^{2}$ CEMSE, King Abdullah University of Science and Technology, Thuwal, SA \\ ${ }^{3}$ Department of Computer Science, George Mason University, VA, USA \\ \{swwei2019, kingjun,gxyu\}@swu.edu.cn, carlotta@cs.gmu.edu, xiangliang.zhang@kaust.edu.sa
}

\begin{abstract}
Multi-view clustering aims at integrating complementary information from multiple heterogeneous views to improve clustering results. Existing multi-view clustering solutions can only output a single clustering of the data. Due to their multiplicity, multi-view data, can have different groupings that are reasonable and interesting from different perspectives. However, how to find multiple, meaningful, and diverse clustering results from multi-view data is still a rarely studied and challenging topic in multi-view clustering and multiple clusterings. In this paper, we introduce a deep matrix factorization based solution (DMClusts) to discover multiple clusterings. DMClusts gradually factorizes multi-view data matrices into representational subspaces layer-by-layer and generates one clustering in each layer. To enforce the diversity between generated clusterings, it minimizes a new redundancy quantification term derived from the proximity between samples in these subspaces. We further introduce an iterative optimization procedure to simultaneously seek multiple clusterings with quality and diversity. Experimental results on benchmark datasets confirm that DMClusts outperforms state-of-the-art multiple clustering solutions.
\end{abstract}

\section{Introduction}

Many real-world data include diverse types of feature views. For example, web images have both visual and textual features; a protein has structure and interactome features. The various feature views embody consistent and complementary information of the same objects, and have produced intensive research in multi-view learning (Bickel and Scheffer 2004; Zhao et al. 2017). The fusion of feature views enables not only the achievement of a comprehensive composite view of the objects, but also facilitates the associated learning task (Nie, Cai, and Li 2017; Tan et al. 2018).

Various efforts have been focused on the development of effective multi-view clustering (MVC) algorithms. Some methods achieve clustering by co-regularization (Kumar and Daumé 2011; Cheng et al. 2013), correlation analysis (Chaudhuri et al. 2009), or multiple kernel learning (Gönen and Alpaydin 2011; Liu et al. 2019); other approaches learn the shared subspace to extract complementary and

\footnotetext{
${ }^{*}$ Corresponding author, kingjun@swu.edu.cn (Jun Wang). Copyright (C) 2020, Association for the Advancement of Artificial Intelligence (www.aaai.org). All rights reserved.
}

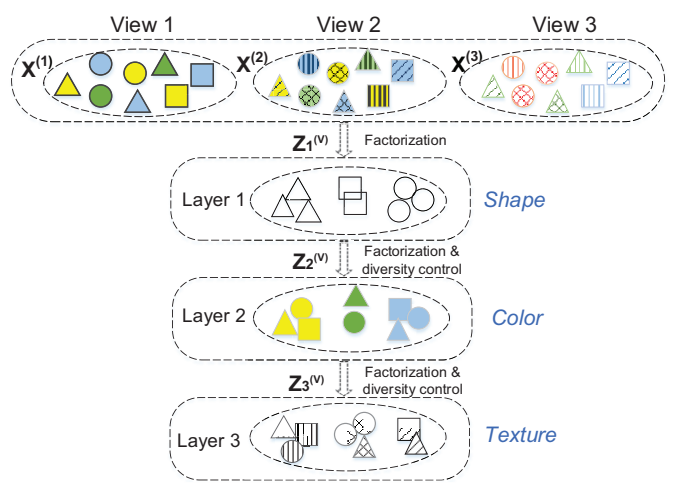

Figure 1: An example of grouping the same objects with three views via deep matrix factorization and diversity control layer-by-layer. The Shape clustering is generated from all three views, while the Color and Texture clusterings are generated from the first two views and the last two views, respectively.

shared information of multi-view data, and perform clustering therein (Li, Jiang, and Zhou 2014; Gao et al. 2015; Zhao, Ding, and Fu 2017; Zong et al. 2017; Kang et al. 2019).

Existing MVC solutions focus on generating a single clustering; they fail to present different but meaningful clusterings of the same multi-view data (Fanaee-T and Thoresen 2018). For example, the three-view objects in Figure 1 have different shapes, colors, and textures. The aforementioned MVC solutions group these objects mainly by shape. But they can also be clustered according to the shared color and texture. These groupings are meaningful but different. In other words, multiple clustering is concerned with both the quality and diversity of alternative clusterings. Although multiple clusterings can present alternative and overlooked meaningful clusterings of the same objects, it is a known dilemma to balance diversity and quality (Bailey 2013). Given this challenge, a number of solutions have been introduced to generate alternative clusterings in different subspaces (Cui, Fern, and Dy 2007; Mautz et al. 2018; Wang et al. 2019), by meta clustering of base clusterings (Caruana et al. 2006), by referring to already explored clusterings (Bae and Bailey 2006; Yang and Zhang 2017), or 
by simultaneously reducing the redundancy between clusterings (Wang et al. 2018; Yao et al. 2019a). However, they still focus on single-view data. One naive extension is to concatenate diverse feature vectors of the same objects into a longer one, and then directly apply off-the-shelf multiple clustering solutions on concatenated vectors. However, this concatenation overrides the intrinsic nature of multi-view data, and thus reduces the quality and increases the redundancy of explored clusterings, as our experiments will show.

To find multiple clusterings on multi-view data, (Yao et al. 2019 b) recently proposed a solution called multi-view multiple clustering (MVMC). MVMC extracts the individual and shared similarity matrices of multi-view data based on the adapted self-representation learning (Luo et al. 2018), and then applies semi-nonnegative matrix factorization (Ding, Li, and Jordan 2010) on each combination of the individual and common similarity data matrices to generate alternative clusterings, where the quality is pursued by the commonality matrix and the diversity is obtained by the individuality matrix. However, MVMC: (a) does not differentiate the relevance of different views and suffers from low-quality (irrelevant) data views; (b) does not maintain well the quality and diversity of multiple clusterings; (c) cannot be applied for datasets with a large number of samples, since it has to factorize the combined similarity matrix with size equal to the number of samples.

In this paper, we introduce a deep matrix factorization based solution (DMClusts, as illustrated in Figure 1) to generate multiple diverse clusterings of good quality in a layerwise fashion. DMClusts collaboratively factorizes the multiview data matrices into multiple representational subspaces layer-by-layer, and seeks an alternative clustering of quality per layer. To achieve diversity among the clusterings, it reduces their redundancy by means of a new balanced redundancy quantification term, which jointly considers the case when two objects are often grouped together and the case when they are in different clusters of the subspaces. We further introduce an iterative optimization procedure to simultaneously seek multiple clusterings in a layer-wise fashion. The main contributions of our work are:

(i) We introduce a deep matrix factorization based solution (DMClusts) to seek multiple clusterings by fusing the consensus and complementary information of multi-view data, and by enforcing the diversity between the clusterings layer-by-layer. DMClusts can credit different degrees of relevance to different views; as such, it's less sensitive to noisy (or low-quality) ones.

(ii) DMClusts introduces a balanced redundancy quantification term, which jointly considers the case that two samples are often nearby in the representational subspace per layer, and the reverse case that they are often faraway per layer, to comprehensively quantify the redundancy of multiple clusterings, whilst existing similar quantification overlooks the latter case. Extensive experiments on benchmark datasets show that DMClusts significantly outperforms other related competitive multiple clusterings solutions (Yao et al. 2019b;
Wang et al. 2019; Yang and Zhang 2017; Ye et al. 2016; Jain, Meka, and Dhillon 2008; Cui, Fern, and Dy 2007) and the deep matrix factorization (Trigeorgis et al. 2017) in finding multiple clusterings with quality and diversity.

\section{Our Method \\ Overview of deep matrix factorization}

Matrix factorization techniques have been extensively adopted for data analysis and representation learning in various domains (Tang et al. 2017; Fu et al. 2018; Li, Tang, and Mei 2019). For example, NMF (nonnegative matrix factorization) (Lee and Seung 2001) can decompose a nonnegative data matrix $\mathbf{X}$ into two factor matrices $\mathbf{X} \approx \mathbf{Z H}$, the nonnegative constraints imposed on factors allow for better interpretability and lead to significantly growing application of NMF and its variants (Ding, Li, and Jordan 2010; Cai et al. 2011; Žitnik and Zupan 2014). By taking $\mathbf{Z} \in$ $\mathbb{R}^{d \times K}$ as $K$ cluster centroids in the $d$-dimensional feature space, and $\mathbf{H} \in \mathbf{R}^{K \times n}$ as the soft membership indicators of $n$ samples to these centroids, semi-NMF (Ding, Li, and Jordan 2010) is equivalent to a soft version of $k$-mean clustering. To absorb mix-sign $\mathbf{X}$, semi-NMF only imposes the nonnegative constraints on $\mathbf{H}$.

To explore the complex hierarchical structure and to eliminate noise in the data matrix $\mathbf{X}$ with different modalities, and motivated by the idea and robustness of deep representation learning (Hinton and Salakhutdinov 2006; Bengio 2009), (Trigeorgis et al. 2017) extends semi-NMF to deep semi-NMF (DMF) as follows:

$$
\begin{aligned}
\mathbf{X} & \approx \mathbf{Z}_{1} \mathbf{H}_{1} \\
\mathbf{X} & \approx \mathbf{Z}_{1} \mathbf{Z}_{2} \mathbf{H}_{2} \\
\quad & \ldots \\
\mathbf{X} & \approx \mathbf{Z}_{1} \mathbf{Z}_{2} \ldots \mathbf{Z}_{m} \mathbf{H}_{m}
\end{aligned}
$$

where $\mathbf{Z}_{l} \in \mathbf{R}^{K_{l-1} \times K_{l}}$ is the $l$-th $(l \leq m)$ layer basis matrix, and $\mathbf{H}_{l} \in \mathbb{R}^{K_{l} \times n}(\geq 0)$ is the $l$-th layer representation matrix. By taking $\mathbf{Z}_{1}, \cdots,\left(\mathbf{Z}_{1} \mathbf{Z}_{2} \cdot \mathbf{Z}_{l}\right) \in \mathbf{R}^{d \times K_{l}}$ as the cluster centroids and $\mathbf{H}_{l} \in \mathbf{R}^{K_{l} \times n}$ as the cluster indicators, or separately clustering on $\mathbf{H}_{l}$, we can obtain $m$ clusterings by a deep factorization network with $m$ layers. However, these clusterings may have high redundancy, since the overlap between them is ignored.

Multi-view data often embody different distributions, which enable different groupings of the same dataset from diverse perspectives. Therefore, it is promising to apply DMF on multi-view data to discover multiple clusterings. One simple solution is to concatenate multiple feature views into a single view, and then directly apply DMF on the concatenated view. However, this concatenation does not differentiate the relevance of these views, and results in information override and redundant clusterings. Given that, we propose the multi-view multiple clusterings using deep matrix factorization solution.

\section{The proposed method}

Suppose $\mathcal{X}=\left\{\mathbf{X}^{(1)}, \mathbf{X}^{(2)}, \cdots, \mathbf{X}^{(V)}\right\}$ is a dataset with $V$ different feature views of $n$ objects, $\mathbf{X}^{(v)} \in \mathbb{R}^{d_{v} \times n}$. To make 
use of the complementary information and to explore hierarchical representations of multi-view data, we formulate our model by extending DMF as follows:

$$
\begin{aligned}
\underset{\mathbf{Z}_{m}^{(v)}, \mathbf{H}_{m}}{\min } & =\sum_{m=1, m^{\prime} \neq m}^{M} \sum_{v=1}^{V}\left\|\mathbf{X}^{(v)}-\mathbf{Z}_{1}^{(v)} \mathbf{Z}_{2}^{(v)} \ldots \mathbf{Z}_{m}^{(v)} \mathbf{H}_{m}\right\|_{F}^{2} \\
& +\lambda \mathcal{R}\left(\mathbf{H}_{m}, \mathbf{H}_{m^{\prime}}\right)
\end{aligned}
$$

where $M$ is the user-specified target number of clusterings, $\mathbf{Z}_{l}^{(v)}$ is the $l$-th $(l \leq m)$ layer mapping for view $v$, $\mathcal{R}\left(\mathbf{H}_{m}, \mathbf{H}_{m^{\prime}}\right)$ quantifies the redundancy between two clusterings and will be discussed later. $\lambda$ is introduced to balance the quality and redundancy of $M$ clusterings. Since $\mathbf{H}_{m}$ is shared across all the data views, we can expect that $\mathbf{H}_{m}$ fuses the complementary information of multiple data views to generate a high-quality representational subspace in the $m$-th layer with respect to $\mathbf{Z}_{1}^{(v)} \mathbf{Z}_{2}^{(v)} \ldots \mathbf{Z}_{m}^{(v)}$. In addition, because of the hierarchical representation and redundancy control term, alternative clusterings with diversity can be pursued also.

Our formulation has a close connection with multi-view clustering via deep matrix factorization (Zhao, Ding, and Fu 2017), which also factorizes multiple data views layer-bylayer to extract the complementary information, but it can only generate a single clustering in the final layer. Our task is different from subspace clustering (Domeniconi et al. 2007; Luo et al. 2018), which seeks only one clustering with different clusters in different subspaces. Our formulation is also different from non-redundant multiple clustering by nonnegative matrix factorization (MNMF) (Yang and Zhang 2017), which performs only one layer factorization to find a new clustering by reducing the redundancy between the clustering and already explored ones. As such, MNMF may generate low quality alternative clusterings due to its one-layer representation of data and the heavy dependence on the reference clustering.

Different data views may have a different relevance toward different clusterings. Eq. (2) and MVMC (Yao et al. $2019 \mathrm{~b}$ ) assume all the data views have the same relevance toward these clusterings. As such, the noisy or irrelevant data views may compromise the quality of alternative clusterings. To account for the different levels of relevance of the data views toward the alternative clusterings, and reduce the impact of noisy views, we further assign weights to these views for each clustering as follows:

$$
\begin{aligned}
& \min _{\mathbf{z}_{l}^{(v)}, \mathbf{H}_{m}, \alpha_{m}^{(v)}}=\sum_{m=1, m^{\prime}>m}^{M} \sum_{v=1}^{V}\left(\alpha_{m}^{(v)}\right)^{r}\left\|\mathbf{X}^{(v)}-\mathbf{Z}_{m}^{(v)} \ldots \mathbf{Z}_{m}^{(v)} \mathbf{H}_{m}\right\|_{F}^{2} \\
&+\lambda \mathcal{R}\left(\mathbf{H}_{m}, \mathbf{H}_{m^{\prime}}\right) \\
& \text { s.t., } \mathbf{H}_{m} \geq 0, \sum_{v=1}^{V} \alpha_{m}^{(v)}=1, \alpha_{m}^{(v)} \geq 0
\end{aligned}
$$

where $\alpha_{m}^{(v)} \geq 0$ is the weight coefficient for the $v$-th data view for generating the $m$-th clustering, and $r$ is the parameter to control the weights distribution. In this way, multiple data views are selectively fused to generate diverse clustering with quality. For example, in Figure 1, three alternative clusterings (shape, color, texture) can be obtained by different weight assignments of three views.

As we stated, it is important to control the redundancy (or overlap) with alternative clusterings. Most subspace based multiple clusterings solutions reduce the redundancy between clusterings by seeking orthogonal (non-redundant or independent) subspaces (Cui, Fern, and Dy 2007; Ye et al. 2016; Mautz et al. 2018; Wang et al. 2019). DMClusts also has such flavor and seeks a clustering based on each layer's representation $\mathbf{H}_{m}$. However, a set of objects maybe nearby in the orthogonally projected subspaces and thus outputs similar clusters in these subspaces. For this reason, we additionally quantify the redundancy between clusterings using $\left(\left\{\mathbf{H}_{m}\right\}_{m=1}^{M}\right)$. A co-association matrix $\mathbf{C}^{(m)} \in \mathbb{R}^{n \times n}$ can reflect whether two objects are grouped into the same cluster or not for the $m$-th clustering (Fred and Jain 2005). Particularly, if $\mathbf{x}_{i}$ and $\mathbf{x}_{j}$ are grouped into the same cluster, then $\mathbf{C}_{i j}^{(m)}=1$, otherwise $\mathbf{C}_{i j}^{(m)}=0$. So if two clusterings ( $m$ and $m^{\prime}$ ) have a large $\sum_{i j}^{n} \mathbf{C}_{i j}^{m} \mathbf{C}_{i j}^{m^{\prime}}$, there is a high redundancy (or overlap) between them. Since the normalized representation $\mathbf{H}_{m}$ often can not be an exact binary cluster-indicator matrix, here we approximate $\mathbf{C}^{(m)}$ by $\mathbf{H}_{m}^{T} \mathbf{H}_{m}$, which softly quantifies the degree of two objects being grouped into the same cluster for the $m$-th layer (or clustering). Based on this approximation, we quantify the overlap between two clusterings in different layers as:

$$
\begin{aligned}
\mathcal{R}\left(\mathbf{H}_{m}, \mathbf{H}_{m^{\prime}}\right) & =\sum_{i, j=1}^{n}\left(\mathbf{H}_{m}^{T} \mathbf{H}_{m}\right)_{i j}\left(\mathbf{H}_{m^{\prime}}^{T} \mathbf{H}_{m^{\prime}}\right)_{i j} \\
& =\operatorname{tr}\left(\mathbf{H}_{m}^{T} \mathbf{H}_{m} \mathbf{H}_{m^{\prime}}^{T} \mathbf{H}_{m^{\prime}}\right)
\end{aligned}
$$

where $\operatorname{tr}(\cdot)$ is the matrix trace operator. A large $\mathcal{R}\left(\mathbf{H}_{m}, \mathbf{H}_{m^{\prime}}\right)$ means $\mathbf{x}_{i}$ and $\mathbf{x}_{j}$ are nearby in different representation subspaces, which will be grouped into the same clusters of two different clusterings and increase the overlap.

However, Eq. (4) only accounts for the case that two objects are often projected nearby (grouped into the same clusters) in different representation subspaces, but overlooks the case that two objects are frequently placed faraway (grouped into different clusters) in these subspaces. We want to remark that other multiple clustering solutions (Yang and Zhang 2017; Wang et al. 2018; Yao et al. 2019a) also adopt the idea in Eq. (4) to quantify the redundancy between clusterings, and thus they also overlook the latter case, which emerges when the number of clusters $\geq 3$. To remedy this overlook, we introduce a balanced redundancy quantification term as follows:

$$
\begin{aligned}
\tilde{\mathcal{R}}\left(\mathbf{H}_{m}, \mathbf{H}_{m^{\prime}}\right) & =\beta \operatorname{tr}\left(\mathbf{H}_{m}^{T} \mathbf{H}_{m} \mathbf{H}_{m^{\prime}}^{T} \mathbf{H}_{m^{\prime}}\right) \\
& +(1-\beta) \operatorname{tr}\left(\left(1-\mathbf{H}_{m}^{T} \mathbf{H}_{m}\right)\left(1-\mathbf{H}_{m^{\prime}}^{T} \mathbf{H}_{m^{\prime}}\right)\right.
\end{aligned}
$$

where $\beta \in[0,1]$ is the balance coefficient. Eq. (5) considers two extreme cases: (i) many pairwise objects are always nearby in two subspaces, (ii) are always faraway in these subspaces. Both cases increase the overlap of two clusterings. In other words, if many pairwise objects placed into 
the same clusters for one clustering, but not so for the other clustering, then the redundancy between them is low.

To this end, we can reformulate the objective function of DMClusts as follows:

$$
\begin{aligned}
\min _{\mathbf{z}_{m}^{(v)}, \mathbf{H}_{m}, \alpha_{m}^{(v)}} \mathcal{J} & =\sum_{m=1, m \neq m^{\prime}}^{M} \sum_{v=1}^{V}\left(\alpha_{m}^{(v)}\right)^{r}\left\|\mathbf{X}^{(v)}-\mathbf{Z}_{1}^{(v)} \ldots \mathbf{Z}_{m}^{(v)} \mathbf{H}_{m}\right\|_{F}^{2} \\
& +\lambda\left(\beta \operatorname{tr}\left(\mathbf{H}_{m}^{T} \mathbf{H}_{m} \mathbf{H}_{m^{\prime}}^{T} \mathbf{H}_{m^{\prime}}\right)\right. \\
& \left.+(1-\beta) \operatorname{tr}\left(\left(1-\mathbf{H}_{m}^{T} \mathbf{H}_{m}\right)^{T}\left(1-\mathbf{H}_{m^{\prime}}^{T} \mathbf{H}_{m^{\prime}}\right)\right)\right) \\
& \text { s.t., } \mathbf{H}_{m} \geq 0, \sum_{v=1}^{V} \alpha_{m}^{(v)}=1, \alpha_{m}^{(v)} \geq 0
\end{aligned}
$$

By minimizing the above objective, we can gradually find $M$ clusterings, while the quality of these clusterings is pursued by the constraint $\mathbf{H}_{m}$ of the respective layer shared across all the views, and the diversity is pursued by reducing the cases that too many objects always nearby (or faraway) in these representation subspaces. Our experiments will confirm the advantage of these factors.

\section{Optimization}

The minimization objective in Eq. (6) is defined with respect to $\mathbf{Z}_{m}^{(v)}, \mathbf{H}_{m}$, and $\alpha_{m}^{(v)}$. Since a close-form solution cannot be given, we alternatively optimize one variable while keeping the other two constant. The alternative process is detailed below.

Update rule for $\mathbf{Z}_{m}^{(v)}$ : The optimization of Eq. (6) with respect to $\mathbf{Z}_{m}^{(v)}$ is:

$$
\mathcal{J}_{Z}\left(\mathbf{Z}_{m}^{(v)}\right)=\sum_{i=m}^{M}\left(\alpha_{i}^{(v)}\right)^{r}\left\|\mathbf{X}^{(v)}-\Phi_{m} \mathbf{Z}_{m}^{(v)} \mathbf{H}_{m i}\right\|_{F}^{2}
$$

where $\Phi_{m}=\mathbf{Z}_{1}^{(v)} \mathbf{Z}_{2}^{(v)} \ldots \mathbf{Z}_{m-1}^{(v)}$ and $\mathbf{H}_{m i}=$ $\mathbf{Z}_{m+1}^{(v)} \ldots \mathbf{Z}_{i}^{(v)} \mathbf{H}_{i}$. Letting the partial derivative $\partial \mathcal{J}_{Z} / \mathbf{Z}_{m}^{(v)}=0$, we can obtain

$$
\begin{gathered}
\mathbf{Z}_{m}^{(v)}=\left(\Phi_{m}^{T} \Phi_{m}\right)^{-1}\left(\sum_{i=m}^{M}\left(\alpha_{i}^{(v)}\right)^{r} \Phi^{T} \mathbf{X}^{(v)} \mathbf{H}_{m i}^{T}\right) \\
\left(\sum_{i=m}^{M}\left(\alpha_{i}^{(v)}\right)^{r} \mathbf{H}_{m i} \mathbf{H}_{m i}^{T}\right)^{-1}
\end{gathered}
$$

Update rule for $\mathbf{H}_{m}$ : Optimizing Eq. (6) with respect to $\mathbf{H}_{m}$ is equivalent to minimizing the following:

$$
\begin{aligned}
& \mathcal{J}_{H}\left(\mathbf{H}_{m}\right)=\sum_{v=1}^{V} \alpha_{m}^{(v)}\left\|\mathbf{X}^{(v)}-\mathbf{Z}_{1}^{(v)} \ldots \mathbf{Z}_{m}^{(v)} \mathbf{H}_{m}\right\|_{F}^{2} \\
&+\lambda\left(\beta \operatorname{tr}\left(\mathbf{H}_{m}^{T} \mathbf{H}_{m} \mathbf{H}_{m^{\prime}}^{T} \mathbf{H}_{m^{\prime}}\right)\right. \\
&\left.+(1-\beta) \operatorname{tr}\left(\left(1-\mathbf{H}_{m}^{T} \mathbf{H}_{m}\right)^{T}\left(1-\mathbf{H}_{m^{\prime}}^{T} \mathbf{H}_{m^{\prime}}\right)\right)\right) \\
& \text { s.t., } \mathbf{H}_{m} \geq 0
\end{aligned}
$$

For the constraint $\mathbf{H}_{m} \geq 0$, we introduce the Lagrangian multiplier $\eta$ as follows:

$$
\begin{aligned}
\mathcal{L}\left(\mathbf{H}_{m}\right) & =\sum_{v=1}^{V} \alpha_{m}^{(v)}\left\|\mathbf{X}^{(v)}-\mathbf{Z}_{1}^{(v)} \ldots \mathbf{Z}_{m}^{(v)} \mathbf{H}_{m}\right\|_{F}^{2} \\
& +\lambda \sum_{m^{\prime}=1, m^{\prime} \neq m}^{M} \beta \operatorname{tr}\left(\mathbf{H}_{m}^{T} \mathbf{H}_{m} \mathbf{H}_{m^{\prime}}^{T} \mathbf{H}_{m^{\prime}}\right) \\
& +(1-\beta) \operatorname{tr}\left(\left(1-\mathbf{H}_{m}^{T} \mathbf{H}_{m}\right)\left(1-\mathbf{H}_{m^{\prime}}^{T} \mathbf{H}_{m^{\prime}}\right)\right)-\operatorname{tr}\left(\eta \mathbf{H}_{m}\right)
\end{aligned}
$$

Letting the partial derivative $\partial \mathcal{L} / \mathbf{H}_{m}=0$ and $\eta_{i j}\left(\mathbf{H}_{m}\right)_{i j}=0$, we can get

$$
\mathbf{H}_{m}=\mathbf{H}_{m} \odot \sqrt{\frac{\mathbf{Q}^{+}+\mathbf{P}^{-} \mathbf{H}_{m}+\lambda \Gamma_{m}^{-}}{\mathbf{Q}^{-}+\mathbf{P}^{+} \mathbf{H}_{m}+\lambda \Gamma_{m}^{+}}}
$$

where $\mathbf{Q}=\sum_{v=1}^{V}\left(\alpha_{m}^{(v)}\right)^{r}\left(\mathbf{Z}_{a l l}^{(v)}\right)^{T} \mathbf{X}^{(v)}, \quad \mathbf{P}=$ $\sum_{v=1}^{V}\left(\alpha_{m}^{(v)}\right)^{r}\left(\mathbf{Z}_{a l l}^{(v)}\right)^{T} \mathbf{Z}_{a l l}^{(v)} \cdot \mathbf{Q}_{i j}^{+}=\left(|\mathbf{Q}|_{i j}+\mathbf{Q}_{i j}\right) / 2, \mathbf{Q}_{i j}^{-}=$ $\left(|\mathbf{Q}|_{i j}-\mathbf{Q}_{i j}\right) / 2, \Gamma_{m}=\sum_{m=1, m^{\prime} \neq m}^{M} \mathbf{H}_{m} \mathbf{H}_{m^{\prime}}^{T} \mathbf{H}_{m^{\prime}}-(1-$ $\beta) \mathbf{H}_{m} \mathbf{1}^{T}, \mathbf{Z}_{\text {all }}^{(v)}=\mathbf{Z}_{1}^{(v)} \mathbf{Z}_{2}^{(v)} \ldots \mathbf{Z}_{m}^{(v)}$.

Update rule for $\alpha_{m}^{(v)}$ : We denote $\Theta_{m}^{(v)}=\| \mathbf{X}^{(v)}-$ $\mathbf{Z}_{1}^{(v)} \ldots \mathbf{Z}_{m}^{(v)} \mathbf{H}_{m} \|_{F}^{2}$. Eq. (6) with respect to $\alpha_{m}^{(v)}$ is written as:

$$
\min _{\alpha_{m}^{(v)}} \sum_{v=1}^{V}\left(\alpha_{m}^{(v)}\right)^{r} \Theta_{m}^{(v)} \text { s.t. } \sum_{v=1}^{V} \alpha_{m}^{(v)}=1, \alpha_{m}^{(v)} \geq 0 .
$$

The Lagrangian function of Eq. (12) is:

$$
\min _{\alpha_{m}^{(v)}} \sum_{v=1}^{V}\left(\alpha_{m}^{(v)}\right)^{r} \Theta_{m}^{(v)}-\lambda\left(\sum_{v=1}^{V} \alpha_{m}^{(v)}-1\right) .
$$

where $\lambda$ is the Lagrangian multiplier. By taking the derivative of Eq. (13) with respect to $\alpha_{m}^{(v)}$, and setting it to zero, we have $\alpha_{m}^{(v)}=\left(\lambda / r \Theta_{m}^{(v)}\right)^{\frac{1}{r-1}}$. Since $\sum_{v=1}^{V}\left(\alpha_{m}^{(v)}\right)^{r}=1$, we can obtain:

$$
\alpha_{m}^{(v)}=\left(r \Theta_{m}^{(v)}\right)^{\frac{1}{1-r}} / \sum_{v=1}^{V}\left(r \Theta_{m}^{(v)}\right)^{\frac{1}{1-r}}
$$

To this end, we have all the iterative update rules for optimizing three variables of DMClusts. We repeat these updates iteratively until convergence. After that, we run $k$-means clustering on each $\left\{\mathbf{H}_{m}\right\}_{m=1}^{M}$ and obtain $M$ clusterings.

\section{Time complexity}

The time complexity of DMClusts is composed of three parts. For simplicity, we assume all the layers have the same size $K$. DMClusts takes order $\mathcal{O}\left(M\left(n d K+d K^{2}+n K^{2}\right)\right)$ to update $\mathbf{Z}_{m}^{(v)}, \mathcal{O}(V n d K)$ to update $\alpha_{m}^{(v)}$, and $\mathcal{O}(V n d K+$ $\left.M n K^{2}\right)$ to update $\mathbf{H}_{m}$ in each iteration. So the time complexity of DMClusts for generating $M$ clusterings on $V$ views is $\mathcal{O}\left(t M\left(M n d K+M n K^{2}+M d K^{2}+V n d K\right)\right)$, where $t$ is the number of iterations to convergence. Generally $K<d, K<n$, and $M \ll n$, thus the complexity of DMClusts is $\mathcal{O}\left(t\left(M^{2}+V\right) n d K\right)$. In our used datasets, DMClusts converges within $t<50$ iterations. On the other hand, the time complexity of MVMC (Yao et al. 2019b) is $\mathcal{O}\left(t V M\left(n^{2} d+n^{2} k\right)\right)$ ( $k$ is the number of clusters). Clearly, the complexity of DMClusts is linear in $n$, but MVMC is quadratic to $n$. As a result, our DMClusts can scale to larger datasets than MVMC. 


\section{Experimental Results and Analysis Experimental Setup}

In this section, we evaluate the effectiveness and efficiency of our proposed DMClusts on seven widely-used multi-view datasets, as described in Table 1. The adopted datasets are from different domains, with different numbers of views and objects. More details on the data are given in the Supplementary file.

Multiple clustering approaches aim to achieve diverse clusterings of high quality. To measure quality, we use Silhouette Coefficient (SC) and the Dunn Index (DI) as internal indexes to quantify the compactness and separation of clusters. To measure redundancy, we use Normalized $\mathrm{Mu}-$ tual Information (NMI) and Jaccard Coefficient (JC) as external indexes to quantify the similarity of clusters between two clusterings. We want to emphasize that a higher value of SC and DI means a clustering with higher quality, but a smaller value of NMI and JC implies that two clusterings have a smaller redundancy. These metrics have been widely adopted for evaluating multiple clusterings (Bailey 2013; Yang and Zhang 2017). Their formal definitions are given in the Supplementary file.

Table 1: Statistics of multi-view datasets. $n, c, V$ are the numbers of objects, clusters and views; $d_{v}$ are the dimensions of $V$ views.

\begin{tabular}{l|l|l}
\hline Datasets & $n, c, V$ & $d_{v}$ \\
\hline Caltech7 & $1474,7,6$ & {$[40,48,254,1984,512,928]$} \\
Handwritten & $2000,10,6$ & {$[216,76,64,6,240,47]$} \\
Reuters & $1200,6,5$ & {$[21531,24892,34251,15506,11547]$} \\
BBCSport & $145,2,4$ & {$[4659,4633,4665,4684]$} \\
MSRCv1 & $210,7,6$ & {$[1302,48,512,100,256,210]$} \\
Yale & $165,15,3$ & {$[4096,3304,6750]$} \\
Mirflickr & $16738,24,2$ & {$[150,500]$} \\
\hline
\end{tabular}

\section{Discovering multiple clusterings}

To comparatively study the performance of DMClusts, we consider Dec-kmeans (Jain, Meka, and Dhillon 2008), MVMC (Yao et al. 2019b), OSC (Cui, Fern, and Dy 2007), ISAAC (Ye et al. 2016), MNMF (Yang and Zhang 2017), and MISC (Wang et al. 2019) as comparing methods. The last four methods use different techniques to seek clusterings in subspaces. The input parameters of the comparing methods are fixed (or optimized) as the authors suggested in their papers or shared code. The input parameters of DMClusts are selected from the following ranges: $r \in\left\{5 \times 10^{-4}, 5 \times 10^{-3}, \ldots, 5\right\}, \lambda \in\left\{10^{-4}, 10^{-3}, \ldots, 10^{4}\right\}$, $\beta \in[0,1]$ and $K_{1} \in\left[k, \min \left(d_{v}\right)\right], K_{2} \in\left[k, K_{1}\right]$ with $M=2$. We fix the number of clusters for each clustering to the number of classes $c$ of each dataset, as reported in Table 1. Existing multiple clustering algorithms (except MVMC and DMClusts) cannot work on multiple view data. Following the solution in (Yao et al. 2019b), we concatenate the feature vectors of multi-view data and then run them on the concatenated vectors to seek alternative clusterings. For reference, we also apply DMF (Trigeorgis et al. 2017) on the concatenated vectors to gradually explore multiple clusterings layer by layer.
MNMF requires input a reference clustering to find an alternative clustering. Here we use $k$-means to generate the reference clustering. For the other comparing methods, we directly use their respective solutions to generate two alternative clusterings $\left(\mathcal{C}_{1}, \mathcal{C}_{2}\right)$. Following the evaluation protocol used by the comparing methods, we measure clustering quality with the average $(\mathrm{SC}$ or $\mathrm{DI})$ of $\mathcal{C}_{1}$ and $\mathcal{C}_{2}$, and we measure the diversity (NMI or JC) between $\mathcal{C}_{1}$ and $\mathcal{C}_{2}$. Table 2 gives the average results of ten independent runs and standard deviations of each method on generating two alternative clusterings. The results of ISAAC and MISC on Reuters and Mirflickr are not reported for their high complexity on large scale datasets.

From Table2, we make the following observations:

(i) Multi-view vs. Concatenated view: Both DMClusts and MVMC directly operate on multi-view data, and their generated two clusterings have a significant lower redundancy than those generated by other comparing methods. In addition, DMClusts frequently obtains a better quality than other comparing methods that can only work on the concatenated view. This shows that the concatenated feature vectors override the intrinsic nature of multi-view data, which help to generate multiple clusterings with diversity. This also expresses the capability of our tailored deep matrix factorization in exploring multiple clusterings with quality.

(ii) DMClusts vs. MVMC: DMClusts generally obtains a significantly better quality (SC and DI) than MVMC, and holds a comparable diversity (NMI and JC). In other words, our DMClusts maintains a better balance of quality and diversity than MVMC. A possible factor is that DMClusts differentiates the relevance of multiple views, whereas MVMC does not. As a result, DMClusts is less sensitive to the noisy views than MVMC. Another factor is that our balanced redundancy term is more comprehensive by considering two types of redundancy, but MVMC considers only one type.

(iii) DMClusts vs. DMF: DMClusts always gives a better performance (both quality and diversity) than DMF, although they both can explore alternative clusterings in a layer-wise fashion. The advantage of DMClusts is two-fold: it accounts for the different relevance of data views, and can selectively fuse them to generate alternative clusterings with quality, while DMF can only operate on the concatenated features without differentiating these views; it also explicitly controls the diversity between alternative clusterings, while DMF does not.

To investigate the robustness of DMClusts to noisy views, we constructed a synthetic dataset on Reuters by injecting a noisy view $\mathbf{X}^{(6)} \in \mathbb{R}^{500 \times 1200}$ following standard Gaussian distribution. We then apply DMF and DMClusts on this synthetic dataset with the input parameters fixed as $r=0.1$, $\lambda=0.1, \beta=0.4$. Next, we visualize the weights assigned to six views for the first and second clusterings in Figure 2. DMClusts indeed assigns different sets of weights to these views for generating two clusterings with a low overlap (NMI: 0.019, JC: 0.161), and it manifests a robustness to the noisy view by assigning it with a zero weight. As a result, DMClusts holds the similar quality and diversity as on the original Reuters. In contrast, DMF has a nearly $50 \%$ reduced quality (SC: 0.158 , DI: 0.015 ) and an about $25 \%$ increased 
Table 2: Quality and Diversity of the various comparing methods on generating multiple clusterings. $\uparrow(\downarrow)$ indicates the preferred direction for the corresponding measure. $\bullet / \circ$ indicates whether our DMClusts is statistically (according to pairwise $t$-test at $95 \%$ significance level) superior/inferior to the other method.

\begin{tabular}{|c|c|c|c|c|c|c|c|c|c|}
\hline & & Dec-kmeans & MNMF & OSC & ISAAC & MISC & MVMC & DMF & DMClusts \\
\hline \multirow{4}{*}{ Caltech7 } & $\mathrm{SC} \uparrow$ & $0.049 \pm 0.002 \bullet$ & $0.234 \pm 0.000 \bullet$ & $0.266 \pm 0.000 \bullet$ & $0.153 \pm 0.010 \bullet$ & $0.201 \pm 0.003 \bullet$ & $0.140 \pm 0.002 \bullet$ & $0.065 \pm 0.011 \bullet$ & $0.301 \pm 0.006$ \\
\hline & $\mathrm{DI} \uparrow$ & $0.042 \pm 0.006 \bullet$ & $0.037 \pm 0.000 \bullet$ & $0.054 \pm 0.000 \bullet$ & $0.027 \pm 0.001 \bullet$ & $0.048 \pm 0.002 \bullet$ & $0.062 \pm 0.000 \bullet$ & $0.065 \pm 0.004 \bullet$ & $0.090 \pm 0.003$ \\
\hline & NMI $\downarrow$ & $0.021 \pm 0.003 \bullet$ & $0.022 \pm 0.000 \bullet$ & $0.693 \pm 0.015 \bullet$ & $0.645 \pm 0.035 \bullet$ & $0.516 \pm 0.015 \bullet$ & $0.006 \pm 0.000 \circ$ & $0.310 \pm 0.035 \bullet$ & $0.009 \pm 0.000$ \\
\hline & $\mathrm{JC} \downarrow$ & $0.127 \pm 0.009 \bullet$ & $0.092 \pm 0.000 \bullet$ & $0.383 \pm 0.000 \bullet$ & $0.358 \pm 0.022 \bullet$ & $0.349 \pm 0.018 \bullet$ & $0.076 \pm 0.000 \circ$ & $0.235 \pm 0.004 \bullet$ & $0.087 \pm 0.002$ \\
\hline \multirow{4}{*}{ BBCSport } & $\mathrm{SC} \uparrow$ & $0.088 \pm 0.007 \bullet$ & $0.014 \pm 0.000 \bullet$ & $0.144 \pm 0.000 \bullet$ & $-0.039 \pm 0.002 \bullet$ & $0.089 \pm 0.002 \bullet$ & $0.269 \pm 0.000 \bullet$ & $0.204 \pm 0.003 \bullet$ & $0.284 \pm 0.006$ \\
\hline & $\mathrm{DI} \uparrow$ & $0.487 \pm 0.001 \circ$ & $0.434 \pm 0.000 \bullet$ & $0.520 \pm 0.000 \circ$ & $0.411 \pm 0.016 \bullet$ & $0.335 \pm 0.009 \bullet$ & $0.014 \pm 0.000 \bullet$ & $0.255 \pm 0.001 \bullet$ & $0.468 \pm 0.010$ \\
\hline & NMI $\downarrow$ & $0.002 \pm 0.000 \bullet$ & $0.086 \pm 0.000 \bullet$ & $0.001 \pm 0.000 \bullet$ & $0.010 \pm 0.001 \bullet$ & $0.009 \pm 0.001 \bullet$ & $0.000 \pm 0.000$ & $0.101 \pm 0.009 \bullet$ & $0.000 \pm 0.000$ \\
\hline & $\mathrm{JC} \downarrow$ & $0.431 \pm 0.030 \bullet$ & $0.392 \pm 0.000 \circ$ & $0.605 \pm 0.000 \bullet$ & $0.495 \pm 0.015 \bullet$ & $0.520 \pm 0.018 \bullet$ & $0.347 \pm 0.000$ 。 & $0.418 \pm 0.001 \bullet$ & $0.401 \pm 0.003$ \\
\hline \multirow{4}{*}{ Handwritten } & $\mathrm{SC} \uparrow$ & $0.050 \pm 0.006 \bullet$ & $0.014 \pm 0.000 \bullet$ & $0.352 \pm 0.000 \bullet$ & $0.235 \pm 0.007 \bullet$ & $0.251 \pm 0.009 \bullet$ & $0.062 \pm 0.000 \bullet$ & $0.034 \pm 0.001 \bullet$ & $0.377 \pm 0.012$ \\
\hline & $\mathrm{DI} \uparrow$ & $0.051 \pm 0.011 \bullet$ & $0.009 \pm 0.000 \bullet$ & $0.107 \pm 0.000 \bullet$ & $0.056 \pm 0.002 \bullet$ & $0.052 \pm 0.003 \bullet$ & $0.083 \pm 0.000 \bullet$ & $0.240 \pm 0.009 \circ$ & $0.159 \pm 0.004$ \\
\hline & NMI $\downarrow$ & $0.070 \pm 0.012 \bullet$ & $0.089 \pm 0.000 \bullet$ & $0.778 \pm 0.000 \bullet$ & $0.712 \pm 0.018 \bullet$ & $0.645 \pm 0.014 \bullet$ & $0.009 \pm 0.000 \circ$ & $0.212 \pm 0.006 \bullet$ & $0.019 \pm 0.001$ \\
\hline & $\mathrm{JC} \downarrow$ & $0.073 \pm 0.003 \bullet$ & $0.078 \pm 0.003 \bullet$ & $0.570 \pm 0.000 \bullet$ & $0.484 \pm 0.016 \bullet$ & $0.414 \pm 0.019 \bullet$ & $0.073 \pm 0.000 \bullet$ & $0.114 \pm 0.003 \bullet$ & $0.066 \pm 0.000$ \\
\hline \multirow{4}{*}{ MSRCv1 } & $\mathrm{SC} \uparrow$ & $-0.062 \pm 0.003 \bullet$ & $-0.193 \pm 0.002 \bullet$ & $0.382 \pm 0.011 \bullet$ & $0.166 \pm 0.003 \bullet$ & $0.331 \pm 0.008 \bullet$ & $0.113 \pm 0.007 \bullet$ & $0.022 \pm 0.001 \bullet$ & $0.556 \pm 0.012$ \\
\hline & $\mathrm{DI} \uparrow$ & $0.043 \pm 0.007 \bullet$ & $0.027 \pm 0.001 \bullet$ & $0.071 \pm 0.007 \bullet$ & $0.012 \pm 0.002 \bullet$ & $0.013 \pm 0.001 \bullet$ & $0.098 \pm 0.003 \bullet$ & $0.277 \pm 0.010 \bullet$ & $0.336 \pm 0.008$ \\
\hline & NMI $\downarrow$ & $0.054 \pm 0.006 \bullet$ & $0.063 \pm 0.005 \bullet$ & $0.736 \pm 0.054 \bullet$ & $0.549 \pm 0.030 \bullet$ & $0.665 \pm 0.017 \bullet$ & $0.053 \pm 0.006 \bullet$ & $50 \pm 0.002 \bullet$ & 0.038 \\
\hline & $\mathrm{JC} \downarrow$ & $0.109 \pm 0.005 \bullet$ & $0.124 \pm 0.003 \bullet$ & $0.519 \pm 0.025 \bullet$ & $0.357 \pm 0.009 \bullet$ & $0.471 \pm 0.020 \bullet$ & $0.078 \pm 0.002 \circ$ & $0.127 \pm 0.005 \bullet$ & $0.087 \pm 0.003$ \\
\hline \multirow{4}{*}{ Yale } & $\mathrm{SC} \uparrow$ & $0.033 \pm 0.002 \bullet$ & $-0.011 \pm 0.001 \bullet$ & $0.221 \pm 0.005 \bullet$ & $-0.020 \pm 0.002 \bullet$ & $-0.066 \pm 0.008 \bullet$ & $-0.045 \pm 0.007 \bullet$ & $0.021 \pm 0.001 \bullet$ & $0.303 \pm 0.019$ \\
\hline & $\mathrm{DI} \uparrow$ & $0.205 \pm 0.014 \bullet$ & $0.114 \pm 0.004 \bullet$ & $0.331 \pm 0.020 \circ$ & $0.076 \pm 0.004 \bullet$ & $0.073 \pm 0.003 \bullet$ & $0.232 \pm 0.012 \bullet$ & $0.285 \pm 0.004 \bullet$ & 0.292 \\
\hline & NMI $\downarrow$ & $0.241 \pm 0.021 \bullet$ & $0.240 \pm 0.007 \bullet$ & $0.812 \pm 0.063 \bullet$ & $0.369 \pm 0.007 \bullet$ & $0.314 \pm 0.009 \bullet$ & $0.251 \pm 0.006 \bullet$ & $0.319 \pm 0.006 \bullet$ & $0.205 \pm 0.004$ \\
\hline & $\mathrm{JC} \downarrow$ & $0.043 \pm 0.002 \bullet$ & $0.066 \pm 0.004 \bullet$ & $0.357 \pm 0.034 \bullet$ & $0.098 \pm 0.003 \bullet$ & $0.091 \pm 0.002 \bullet$ & $0.055 \pm 0.001 \bullet$ & $0.098 \pm 0.005 \bullet$ & $0.038 \pm 0.002$ \\
\hline \multirow{4}{*}{ Reuters } & $\mathrm{SC} \uparrow$ & $-0.002 \pm 0.000 \bullet$ & $-0.107 \pm 0.009 \bullet$ & $0.065 \pm 0.000 \bullet$ & - & - & $0.180 \pm 0.000 \bullet$ & $0.314 \pm 0.004 \bullet$ & $0.344 \pm 0.006$ \\
\hline & $\mathrm{DI} \uparrow$ & $0.157 \pm 0.008 \circ$ & $0.070 \pm 0.003 \bullet$ & $0.210 \pm 0.000 \circ$ & - & - & $0.038 \pm 0.000 \bullet$ & $0.028 \pm 0.001 \bullet$ & $0.136 \pm 0.005$ \\
\hline & NMI $\downarrow$ & $0.041 \pm 0.004 \bullet$ & $0.033 \pm 0.010 \bullet$ & $0.491 \pm 0.000 \bullet$ & - & - & $0.004 \pm 0.000 \circ$ & $0.508 \pm 0.005 \bullet$ & $0.018 \pm 0.000$ \\
\hline & $\mathrm{JC} \downarrow$ & $0.199 \pm 0.005 \bullet$ & $0.148 \pm 0.002 \bullet$ & $0.454 \pm 0.000 \bullet$ & - & - & $0.091 \pm 0.000 \circ$ & $0.590 \pm 0.011 \bullet$ & $0.132 \pm 0.003$ \\
\hline \multirow{4}{*}{ Mirflicker } & $\mathrm{SC} \uparrow$ & $-0.004 \pm 0.000 \bullet$ & $-0.058 \pm 0.000 \bullet$ & $0.017 \pm 0.000 \bullet$ & - & - & $-0.038 \pm 0.000 \bullet$ & $0.005 \pm 0.000 \bullet$ & $0.336 \pm 0.008$ \\
\hline & $\mathrm{DI} \uparrow$ & $0.061 \pm 0.002 \bullet$ & $0.053 \pm 0.001 \bullet$ & $0.059 \pm 0.002 \bullet$ & - & - & $0.173 \pm 0.005 \circ$ & $0.027 \pm 0.001 \bullet$ & $0.076 \pm 0.001$ \\
\hline & NMI $\downarrow$ & $0.427 \pm 0.012 \bullet$ & $0.014 \pm 0.000 \bullet$ & $0.575 \pm 0.011 \bullet$ & - & - & $0.005 \pm 0.000 \circ$ & $0.108 \pm 0.003 \bullet$ & $0.043 \pm 0.001$ \\
\hline & $\mathrm{JC} \downarrow$ & $0.878 \pm 0.022 \bullet$ & $0.023 \pm 0.000 \circ$ & $0.368 \pm 0.011 \bullet$ & 一 & 一 & $0.022 \pm 0.000 \circ$ & $0.049 \pm 0.001 \bullet$ & $0.033 \pm 0.001$ \\
\hline
\end{tabular}

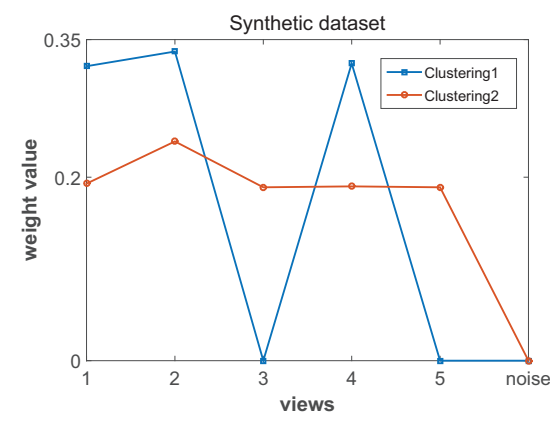

Figure 2: DMClusts assigns two sets of weights to six views for generating two clusterings. The 6-th view is a noisy view.

diversity (NMI: 0.471, JC: 0.375). The increase in diversity is obtained at the expense of a reduced quality. Nevertheless, DMClusts still gives a better diversity than DMF. This investigation corroborates the benefit of weighting views.

To further study whether DMClusts can generate $M \geq 3$ clusterings, we fix the number of target clusterings to $M=4$ and the number clusters for each clustering to $k=3$. Next, we apply DMClusts, DMF, and MVMC on the Handwritten dataset with images in 10 digits and visualize their clusterings in Figure 3. Each row of the subfigure represents a clustering and each image corresponds to the mean of the cluster. The numbers under each image are the dominant digits (not all) in the cluster. It is well known that the handwritten 10 digits are ambiguous and resemble different numbers (7 alike 4 and 3; 9 alike 5 and 7). As such, there is a tendency to group them together in different alternative clusterings. Due to the use of diversity control, DMClusts presents four clusterings without any completely overlapping clusters. In contrast, DMF does not account for diversity and generates some largely overlapping clusters (i.e., $\{0,1,3\},\{2,4,7\}$ in $\mathcal{C}_{3}$ and $\mathcal{C}_{4}$ ). Although MVMC also quantifies the redundancy of two objects often grouped into the same cluster of different clusterings, it still generates a heavily overlapping cluster $\{1,2,3\}$ in $\mathcal{C}_{1}$ and $\mathcal{C}_{3}$. This visual example not only confirms the effectiveness of DMClusts in generating multiple diverse clusterings, but also proves the effectiveness of our balanced redundancy quantification.

\section{Parameter analysis}

Several input parameters $(\lambda, r$ and $\beta)$ may affect the performance of DMClusts. $\lambda$ balances the importance of deep matrix factorization and the diversity control term, $r$ controls the weight distribution assigned to input views, $\beta$ balances the redundancy of two objects placed into the same clusters and redundancy of two objects placed into different clusters of two clusterings.

We study the impact of $\lambda$ by varying it from $10^{-4}$ to $10^{4}$, and plot the change of Quality (DI) and Diversity (1-NMI, the larger the better) of DMClusts on the Yale dataset in Figure 4a with $r=0.5, \beta=0.4$. We find that: (i) diversity (1NMI) steadily increases at first but not so when $\lambda \geq 1$; (ii) the quality (DI) gradually decreases as $\lambda$ increases, and becomes relatively stable after $\lambda \geq 1$. This pattern is explainable, since a larger $\lambda$ forces DMClusts to focus more on the diversity between clusterings, and thus may drag down the quality of the respective clusterings. Overall, this observation confirms the dilemma between diversity and quality of multiple clusterings, and shows the necessity of introducing $\lambda$ to control the redundancy.

We investigate the impact of $r$ by varying it in the grid of $\left\{5 \times 10^{-4}, 5 \times 10^{-3}, \cdots, 5\right\}$, and report the quality and diversity of DMClusts on the Yale dataset in Figure $4 \mathrm{~b}$ with $\lambda=0.01, \beta=0.4$. The quality slightly rises as $r$ increases, 


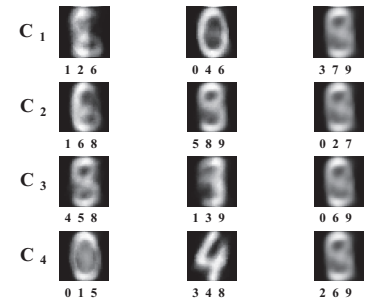

(a) DMClusts

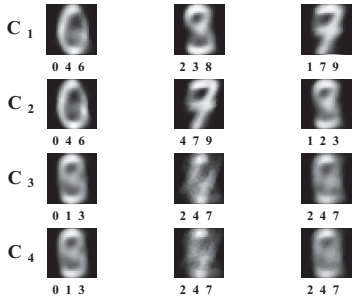

(b) DMF

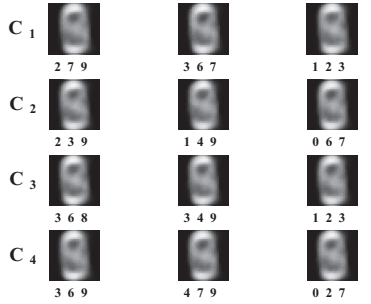

(c) MVMC

Figure 3: Four alternative clusterings $\left(\mathcal{C}_{1}\right.$ to $\left.\mathcal{C}_{4}\right)$ generated by DMClusts (a), DMF (b) and MVMC (c).

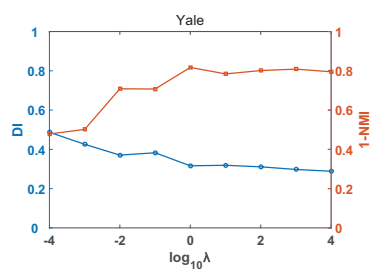

(a) DI and (1-NMI) vs. $\lambda$

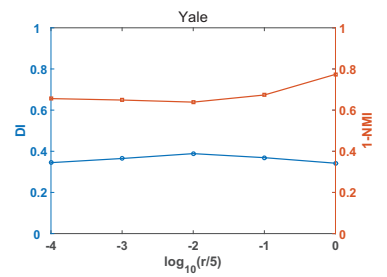

(b) DI and (1-NMI) vs. $r$

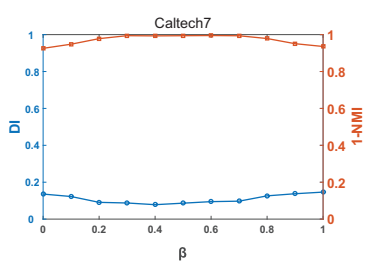

(c) DI and (1-NMI) vs. $\beta$

Figure 4: Quality (DI) and Diversity (1-NMI) of DMClusts vs. $\lambda, r$ and $\beta$.

and the diversity remains stable. When $r>0.05$, the diversity steadily increases and the quality gradually decreases, due to the increased diversity and the known trade-off between diversity and quality. This is because a too small $r$ gives nearly equal weights to all the views, while a moderate $r$ can assign different sets of weights to these views, which helps to generate diverse clusterings, as exampled in Figure 1.

To study the benefit of our balanced redundancy quantification term, we vary $\beta$ from 0 to 1 and report the results in Figure $4 \mathrm{c}$ with $r=0.5, \lambda=0.01$. We observe that the diversity (1-NMI) increases as $\beta$ increase but turns to reduce as $\beta>0.7$. Due to the dilemma between quality and diversity, the quality shows a reverse trend. Neither $\beta=1$ nor $\beta=0$ gives the highest diversity, and $\beta \in[0.3,0.7]$ gives an $\mathrm{NMI} \approx 0$. This observation proves the contribution of considering the previously overlooked redundancy due to two samples placed in different clusters of two clusterings, and also justifies the effectiveness of our balanced redundancy quantification term. In addition, it clarifies why our DMClusts obtains a better diversity between clusterings. We observe that $\beta=1$ (NMI: 0.064 ) gives a larger diversity (by $\approx 16 \%$ ) than $\beta=0$ (NMI: 0.075). This suggests the redundancy two samples in the same clusters of two clusterings is more important than the redundancy they in different clusters. Overall, these two types of redundancy complement each other and help to generate multiple clusterings with improved diversity.

We further study the impact of the number of clusters, layer size $K_{l}$ and of different $M$. We also make a runtime experiment and show that DMClusts not only outperforms the state-of-the-art methods in exploring multiple clusterings with quality and diversity, but also holds a moderate efficiency. The results and analysis as well as convergence analysis can be found in the Supplementary file. Finally, we want to remark that, all the four metrics do not depend on the ground-truth labels of the tested dataset, so the suitable values for parameters can be chosen based on the user's preference toward quality or diversity.

\section{Conclusion}

In this paper, we introduce DMClusts to explore multiple clusterings from multi-view data, which is an interesting, practical but overlooked clustering topic that conjoins multiview clusterings and multiple clusterings. DMClusts adapts the deep matrix factorization to a deep learning approach, and introduces a novel balanced diversity quantification term to seek multiple diverse clusterings of quality. DMClusts shows a superior effectiveness and efficiency than state-ofthe-art competitive solutions. We will investigate a principle to determine a suitable number of layers (clusterings).

\section{Acknowledgments}

This work is supported by NSFC (61872300 and 61873214), Fundamental Research Funds for the Central Universities (XDJK2019B024), Natural Science Foundation of CQ CSTC (cstc2018jcyjAX0228) and by the King Abdullah University of Science and Technology (KAUST), Saudi Arabia. The code and Supplementary file of DMClusts is available at http://mlda.swu.edu.cn/codes.php?name=DMClusts .

\section{References}

Bae, E., and Bailey, J. 2006. Coala: A novel approach for the extraction of an alternate clustering of high quality and high dissimilarity. In ICDM, 53-62. 
Bailey, J. 2013. Alternative clustering analysis: A review. In Charu, A., and Chandan, R., eds., Data Clustering: Algorithms and Applications. CRC Press. 535-550.

Bengio, Y. 2009. Learning deep architectures for ai. Foundations and Trends $\mathbb{R}$ in Machine Learning 2(1):1-127.

Bickel, S., and Scheffer, T. 2004. Multi-view clustering. In ICDM, 19-26.

Cai, D.; He, X.; Han, J.; and Huang, T. S. 2011. Graph regularized nonnegative matrix factorization for data representation. TPAMI 33(8):1548-1560.

Caruana, R.; Elhawary, M.; Nguyen, N.; and Smith, C. 2006. Meta clustering. In ICDM, 107-118.

Chaudhuri, K.; Kakade, S. M.; Livescu, K.; and Sridharan, K. 2009. Multi-view clustering via canonical correlation analysis. In $I C M L, 129-136$.

Cheng, W.; Zhang, X.; Guo, Z.; Wu, Y.; Sullivan, P. F.; and Wang, W. 2013. Flexible and robust co-regularized multidomain graph clustering. In $K D D, 320-328$.

Cui, Y.; Fern, X. Z.; and Dy, J. G. 2007. Non-redundant multi-view clustering via orthogonalization. In ICDM, 133142.

Ding, C. H.; Li, T.; and Jordan, M. I. 2010. Convex and semi-nonnegative matrix factorizations. TPAMI 32(1):4555.

Domeniconi, C.; Gunopulos, D.; Ma, S.; Yan, B.; AlRazgan, M.; and Papadopoulos, D. 2007. Locally adaptive metrics for clustering high dimensional data. DAMI 14(1):63-97.

Fanaee-T, H., and Thoresen, M. 2018. Multi-insight visualization of multi-omics data via ensemble dimension reduction and tensor factorization. Bioinformatics 35(10):16251633.

Fred, A. L., and Jain, A. K. 2005. Combining multiple clusterings using evidence accumulation. TPAMI 27(6):835850.

Fu, G.; Wang, J.; Domeniconi, C.; and Yu, G. 2018. Matrix factorization-based data fusion for the prediction of lncrnadisease associations. Bioinformatics 34(9):1529-1537.

Gao, H.; Nie, F.; Li, X.; and Huang, H. 2015. Multi-view subspace clustering. In ICCV, 4238-4246.

Gönen, M., and Alpaydın, E. 2011. Multiple kernel learning algorithms. JMLR 12(7):2211-2268.

Hinton, G. E., and Salakhutdinov, R. R. 2006. Reducing the dimensionality of data with neural networks. Science 313(5786):504-507.

Jain, P.; Meka, R.; and Dhillon, I. S. 2008. Simultaneous unsupervised learning of disparate clusterings. Statistical Analysis and Data Mining 1(3):195-210.

Kang, Z.; Guo, Z.; Huang, S.; Wang, S.; Chen, W.; Su, Y.; and Xu, Z. 2019. Multiple partitions aligned clustering. In IJCAI, 2701-2707.

Kumar, A., and Daumé, H. 2011. A co-training approach for multi-view spectral clustering. In ICML, 393-400.

Lee, D. D., and Seung, H. S. 2001. Algorithms for nonnegative matrix factorization. In NeurIPS, 556-562.
Li, S.-Y.; Jiang, Y.; and Zhou, Z.-H. 2014. Partial multi-view clustering. In AAAI, 1968-1974.

Li, Z.; Tang, J.; and Mei, T. 2019. Deep collaborative embedding for social image understanding. TPAMI 41(9):2070-2083.

Liu, X.; Zhu, X.; Li, M.; Wang, L.; Zhu, E.; Liu, T.; Kloft, M.; Shen, D.; Yin, J.; and Gao, W. 2019. Multiple kernel k-means with incomplete kernels. TPAMI 99(1):1-14.

Luo, S.; Zhang, C.; Zhang, W.; and Cao, X. 2018. Consistent and specific multi-view subspace clustering. In AAAI, 37303737.

Mautz, D.; Ye, W.; Plant, C.; and Böhm, C. 2018. Discovering non-redundant k-means clusterings in optimal subspaces. In KDD, 1973-1982.

Nie, F.; Cai, G.; and Li, X. 2017. Multi-view clustering and semi-supervised classification with adaptive neighbours. In AAAI, 2408-2414.

Tan, Q.; Yu, G.; Domeniconi, C.; Wang, J.; and Zhang, Z. 2018. Incomplete multi-view weak-label learning. In IJCAI, 2703-2709.

Tang, J.; Shu, X.; Qi, G.-J.; Li, Z.; Wang, M.; Yan, S.; and Jain, R. 2017. Tri-clustered tensor completion for socialaware image tag refinement. TPAMI 39(8):1662-1674.

Trigeorgis, G.; Bousmalis, K.; Zafeiriou, S.; and Schuller, B. W. 2017. A deep matrix factorization method for learning attribute representations. TPAMI 39(3):417-429.

Wang, X.; Yu, G.; Domeniconi, C.; Wang, J.; Yu, Z.; and Zhang, Z. 2018. Multiple co-clusterings. In ICDM, 13081313.

Wang, X.; Wang, J.; Yu, G.; Domeniconi, C.; Xiao, G.; and Guo, M. 2019. Multiple independent subspace clusterings. In AAAI, 5353-5360.

Yang, S., and Zhang, L. 2017. Non-redundant multiple clustering by nonnegative matrix factorization. Machine Learning 106(5):695-712.

Yao, S.; Yu, G.; Wang, X.; Wang, J.; Domeniconi, C.; and Guo, M. 2019a. Discovering multiple co-clusterings in subspaces,. In SDM, 423-431.

Yao, S.; Yu, G.; Wang, J.; Domeniconi, C.; and Zhang, X. 2019b. Multi-view multiple clustering. In IJCAI, 41214127.

Ye, W.; Maurus, S.; Hubig, N.; and Plant, C. 2016. Generalized independent subspace clustering. In ICDM, 569-578.

Zhao, J.; Xie, X.; Xu, X.; and Sun, S. 2017. Multi-view learning overview: recent progress and new challenges. Information Fusion 38:43-54.

Zhao, H.; Ding, Z.; and Fu, Y. 2017. Multi-view clustering via deep matrix factorization. In AAAI, 2921-2927.

Žitnik, M., and Zupan, B. 2014. Data fusion by matrix factorization. TPAMI 37(1):41-53.

Zong, L.; Zhang, X.; Zhao, L.; Yu, H.; and Zhao, Q. 2017. Multi-view clustering via multi-manifold regularized nonnegative matrix factorization. Neural Networks 88:74-89. 